\title{
Multiregular Point Systems
}

\author{
N. P. Dolbilin, ${ }^{1}$ J. C. Lagarias, ${ }^{2}$ and M. Senechal ${ }^{3}$ \\ ${ }^{1}$ Steklov Mathematical Institute, \\ Russian Academy of Sciences, \\ 42 Vavilov St., \\ 117466 Moscow, Russia \\ nikolai@dolbilin.mian.su \\ ${ }^{2}$ Room 2C-373, \\ AT\&T Labs-Research, \\ 180 Park Avenue, \\ Florham Park, NJ 07932-0971, USA \\ jcl@ research.att.com \\ ${ }^{3}$ Department of Mathematics, \\ Smith College, \\ Northampton, MA 01056, USA \\ senechal@minkowski.smith.edu
}

\begin{abstract}
This paper gives several conditions in geometric crystallography which force a structure $X$ in $\mathbb{R}^{n}$ to be an ideal crystal. An ideal crystal in $\mathbb{R}^{n}$ is a finite union of translates of a full-dimensional lattice. An $(r, R)$-set is a discrete set $X$ in $\mathbb{R}^{n}$ such that each open ball of radius $r$ contains at most one point of $X$ and each closed ball of radius $R$ contains at least one point of $X$. A multiregular point system $X$ is an $(r, R)$-set whose points are partitioned into finitely many orbits under the symmetry group $\operatorname{Sym}(X)$ of isometries of $\mathbb{R}^{n}$ that leave $X$ invariant. Every multiregular point system is an ideal crystal and vice versa. We present two different types of geometric conditions on a set $X$ that imply that it is a multiregular point system. The first is that if $X$ "looks the same" when viewed from $n+2$ points $\left\{\mathbf{y}_{i}: 1 \leq i \leq n+2\right\}$, such that one of these points is in the interior of the convex hull of all the others, then $X$ is a multiregular point system. The second is a "local rules" condition, which asserts that if $X$ is an $(r, R)$-set and all neighborhoods of $X$ within distance $\rho$ of each $\mathbf{x} \in X$ are isometric to one of $k$ given point configurations, and $\rho$ exceeds $C R k$ for $C=2\left(n^{2}+1\right) \log _{2}(2 R / r+2)$, then $X$ is a multiregular point system that has at most $k$ orbits under the action of $\operatorname{Sym}(X)$ on $\mathbb{R}^{n}$. In particular, ideal crystals have perfect local rules under isometries.
\end{abstract}




\section{Introduction}

One of the central problems in long-range order is to characterize the various types of ordered discrete structures in $\mathbb{R}^{n}$ that are possible. The symmetry group $\operatorname{Sym}(X)$ of a discrete set $X$ is the set of all Euclidean isometries of $\mathbb{R}^{n}$ that map $X$ to itself. Crystalline materials have the highest possible symmetry. An ideal crystal or perfect crystal is a discrete set $X$ in $\mathbb{R}^{n}$ which is a finite union of translates of a full-dimensional lattice $L$ in $\mathbb{R}^{n}$. An ideal crystal is just a discrete set $X$ that contains a full-dimensional lattice $L$ in its symmetry group; however, $\operatorname{Sym}(X)$ need not act transitively on $X$. This definition of ideal crystal extends a definition of a three-dimensional crystal composed of several species of atoms that was proposed in 1888 by Sohncke [29, p. 433].

The recent discovery and study of quasicrystals (see [25]) raises the question: What geometric and physical conditions force a structure to be crystalline rather than quasicrystalline? In this paper we prove several results giving minimal geometric conditions on a discrete set $X$ in $\mathbb{R}^{n}$ that are sufficient to imply that $X$ is an ideal crystal. These conditions involve concepts in geometric crystallography, and particularly the notion of multiregular point systems which we define below.

Geometric crystallography was initiated by Bravais [1] and Sohncke [27]-[29], see also [9], [14, Chapter 2], and [24]. The basic structures that it studies are Delone sets, also called Delaunay sets or $(r, R)$-sets, which are those sets $X$ in $\mathbb{R}^{n}$ that satisfy the two conditions:

(i) Uniform discreteness. There is an $r>0$ such that any open ball of radius $r$ in $\mathbb{R}^{n}$ contains at most one point of $X$.

(ii) Relative denseness. There is an $R>0$ such that any closed ball of radius $R$ in $\mathbb{R}^{n}$ contains at least one point of $X$.

These sets are named after B. N. Delone who introduced them in 1937, see [6] and [12].

Geometric crystallography is concerned with how a Delone set $X$ looks when viewed from each of its points. A regular point system $X$ is a Delone set such that $X$ "looks the same" from every point $\mathbf{x} \in X$. Regular point systems $X$ are exactly those Delone sets whose symmetry group $\operatorname{Sym}(X)$ acts transitively on $X$, so that $X$ is a single orbit $\operatorname{Sym}(X) \cdot \mathbf{x}$ of $\operatorname{Sym}(X)$. Alternatively, we define the (global) star $\operatorname{ST}_{\mathbf{y}}(X)$ of a set $X$ centered at a point $\mathbf{y} \in \mathbb{R}^{n}$ to be the collection of line segments from $\mathbf{y}$ to every $\mathbf{x} \in X$, namely

$$
\operatorname{ST}_{\mathbf{y}}(X):=\{[\mathbf{y}, \mathbf{x}]: \mathbf{x} \in X\} .
$$

Then $X$ is a regular point system if for each pair $\mathbf{x}_{1}, \mathbf{x}_{2} \in X$ there exists an isometry $g=g\left(\mathbf{x}_{1}, \mathbf{x}_{2}\right)$ such that

$$
g\left(\operatorname{ST}_{\mathbf{x}_{1}}(X)\right)=\operatorname{ST}_{\mathbf{x}_{2}}(X) .
$$

We set

$$
\operatorname{ST}(X):=\left\{\operatorname{ST}_{\mathbf{x}}(X): \mathbf{x} \in X\right\},
$$

and let $|\mathrm{ST}(X)|$ denote the number of distinct isometry classes among all $\mathrm{ST}_{\mathbf{x}}(X)$ for $\mathbf{x} \in X$. A regular point system is just a Delone set $X$ with the property that $|\mathrm{ST}(X)|=1$.

One of the major results of geometric crystallography is that a regular point system is an ideal crystal. However not all ideal crystals are regular point systems. 
Definition 1.1. A multiregular point system is any Delone set $X$ such that the action of its symmetry group $\operatorname{Sym}(X)$ partitions the points of $X$ into a finite number of orbits. That is, a multiregular point system is any Delone set $X$ such that $|\mathrm{ST}(X)|$ is finite.

Each ideal crystal is a multiregular point system. In fact, multiregular point systems coincide with ideal crystals, as was shown by Galiulin [11]. The following theorem includes this result, and also gives another characterization of multiregular point systems, in terms of the concept of crystallographic group, for which we give several equivalent definitions in Section 2.

Theorem 1.1. The following conditions on a set $X$ in $\mathbb{R}^{n}$ are equivalent:

(i) $X$ is a multiregular point system. That is, $X$ is a Delone set and the set of isometry classes among $\left\{\mathrm{ST}_{\mathbf{x}}(X): \mathbf{x} \in X\right\}$ is finite.

(ii) $X$ is an ideal crystal. That is, $X$ is a finite union of translates of an $n$-dimensional lattice $L$, with $X=\bigcup_{i=1}^{k}\left(\mathbf{x}_{i}+L\right)$.

(iii) $X$ is a discrete set whose symmetry group $\operatorname{Sym}(X)$ is a crystallographic group.

The equivalence (ii) $\Leftrightarrow$ (iii) is a well-known corollary of Bieberbach's first theorem (see [31, Theorem 2]). We give a proof of Theorem 1.1 at the end of Section 2.

The first type of geometric condition on a set $X$ that we consider requires that suitable finite sets of global stars $\operatorname{ST}_{\mathbf{y}_{i}}(X)$ of $X$ be isometric. These conditions are global in the sense that a star involves lines to every point of $X$; however, only finitely many stars are used. We show:

Theorem 1.2. Let $X$ be a discrete set in $\mathbb{R}^{n}$ and let $Y$ be a finite set in $\mathbb{R}^{n}$ with $|Y|=n+2$, such that the convex hull of $Y$ is a full-dimensional simplex containing one point of $Y$ in its interior. If the $n+2$ stars $\left\{\mathrm{ST}_{\mathbf{y}}(X): \mathbf{y} \in Y\right\}$ are all isometric, then $\operatorname{Sym}(X)$ is a crystallographic group, and $X$ is an ideal crystal.

It is easy to show that every ideal crystal contains a set $Y \subseteq X$ having the properties above. We derive Theorem 1.2 in Section 3 as a special case of a more general criterion (Theorem 3.1).

The condition on $Y$ in Theorem 1.2 cannot be relaxed to merely requiring that the convex hull of $Y$ be full dimensional. For example, let $X$ in $\mathbb{R}^{3}$ be the 12 vertices of a regular icosahedron, and take $Y=X$. Here $\operatorname{Sym}(X)$ is the alternating group $A_{5}$, which is transitive on $X$, and all $\left\{\operatorname{ST}_{\mathbf{x}}(X): \mathbf{x} \in X\right\}$ are isometric, but $X$ is not an ideal crystal.

The second type of geometric condition that we consider consists of "local rules" that require that the elements in $X$ in a finite neighborhood of each $\mathbf{x} \in X$ of fixed radius be isometric to one of a finite set of point configurations.

Definition 1.2. A set of local rules of radius $\rho_{0}$ is a finite collection $\mathcal{L}=\left\{L_{i}\right\}$ of discrete sets $L_{i}$ with $\mathbf{0} \in L_{i}$, and with each $L_{i}$ contained in the closed ball $B\left(\mathbf{0} ; \rho_{0}\right)$ of radius $\rho_{0}$ around $\mathbf{0}$. A set $X$ satisfies the local rules $\mathcal{L}$ under isometries if for each $\mathbf{x} \in X$ the set $(X-\mathbf{x}) \cap B\left(\mathbf{0} ; \rho_{0}\right)$ is a rotation of some set $L_{i}$ in $\mathcal{L}$. 
In 1976 Delone et al. [6] showed that if $X$ is an $(r, R)$-set in $\mathbb{R}^{n}$ such that all neighborhoods of $X$ within a distance $\rho_{0}=\rho_{0}(r, R, n)$ of each point $\mathbf{x} \in X$ are isometric, then $X$ is a regular point system. In 1988 Dolbilin and Shtogrin [8] announced an analogous result for multiregular point systems. We prove here such a result for multiregular point systems and give an explicit distance bound $\rho_{0}(r, R, n, k)$, where $k=|\mathrm{ST}(X)|$, given by (1.4) below.

Definition 1.3. Given $\rho \in \mathbb{R}_{>0}$, the $\rho$-star of $X$ centered at $\mathbf{y} \in \mathbb{R}^{n}$ is the set of line segments

$$
\operatorname{ST}_{\mathbf{y}}(X ; \rho):=\{[\mathbf{x}, \mathbf{y}]: \mathbf{x} \in X \text { and }\|\mathbf{x}-\mathbf{y}\| \leq \rho\}
$$

The set of all $\rho$-stars of $X$ is denoted

$$
\operatorname{ST}(X ; \rho):=\left\{\operatorname{ST}_{\mathbf{x}}(X ; \rho): \mathbf{x} \in X\right\} .
$$

We let $|\mathrm{ST}(X ; \rho)|$ denote the number of isometry classes among the $\operatorname{ST}_{\mathbf{x}}(X ; \rho)$ for $\mathbf{x} \in X$, and define the function

$$
N_{X}(\rho):=|\operatorname{ST}(X ; \rho)|, \quad 0<\rho<\infty .
$$

In Section 4 we prove:

Theorem 1.3. Let $X$ be a Delone set in $\mathbb{R}^{n}$ with constants $(r, R)$. If for some radius $\rho$ the number $k=N_{X}(\rho)$ of isometry classes of its $\rho$-stars satisfies

$$
N_{X}(\rho)<\frac{\rho}{C R}
$$

with

$$
C=2\left(n^{2}+1\right) \log _{2}\left(\frac{2 R}{r}+2\right),
$$

then $X$ is a multiregular point system, and the global stars $\left\{\operatorname{ST}_{\mathbf{x}}(X): \mathbf{x} \in X\right\}$ of $X$ are partitioned into exactly $k$ orbits under the action of $\operatorname{Sym}(X)$. Furthermore, the only Delone sets $Y$ all of whose $\rho$-stars are isometric to $\rho$-stars of $X$ are sets globally isometric to $X$, i.e.,

$$
Y=Q(X+\mathbf{t}) \quad \text { for some } \quad \mathbf{t} \in \mathbb{R}^{n}, \quad Q \in O(n, \mathbb{R})
$$

The bound (1.1) in Theorem 1.3 is best possible up to the value of $C$, for in Section 4 we give examples of $(r, R)$-sets $X$ in $\mathbb{R}^{n}$ which are not multiregular point systems, but have the property that $N_{X}(\rho)$ is approximately $(\sqrt{n} / 2) \rho / R$ for all large $\rho$.

The interest of Theorem 1.3 to crystallography is that it asserts that the atomic structure of any ideal crystal is completely determined by "local conditions" of a fixed radius around each atom separately.

Definition 1.4. Two sets $X$ and $X^{\prime}$ are locally isomorphic if for each finite neighborhood in $X$ an isometric copy of it can be found in $X^{\prime}$, and vice versa. 
Definition 1.5. A set of local rules $\mathcal{L}$ are perfect local rules under isometries if whenever two sets $X$ and $X^{\prime}$ satisfy $\mathcal{L}$, then $X$ and $X^{\prime}$ are locally isomorphic. That is, $\mathcal{L}$ determines a unique local isomorphism class of sets.

If $X$ is a multiregular point system with parameters $(r, R)$ and with $k$ orbits under the action of $\operatorname{Sym}(X)$, then the set $\mathcal{L}$ of $\rho_{0}$-stars of $X$ for any

$$
\rho_{0}>C R k
$$

is a set of perfect local rules under isometries for $X$. Indeed (1.3) then holds and Theorem 1.3 shows that the local isomorphism class of $X$ consists only of sets isometric to $X$. Thus every ideal crystal satisfies perfect local rules under isometries.

There are also various "quasicrystalline" structures that have perfect local rules under isometries. For further discussions and references concerning "perfect local rules" for ideal crystals and quasicrystals, see [15], [17], [19], and [25].

One question remaining about Theorem 1.3 is the extent to which the bound for $C$ in (1.3) can be improved. The value of $C$ in (1.3) depends on $n, R$, and $r$. It is possible that there exists a smaller bound for $C$ which is a function of the dimension alone. Shtogrin [26] showed for $n=2$ and $k=1$ that $\rho>4 R$ suffices for $X$ to be a multiregular point system, and gave an example showing that $4 R$ is the best possible value. Dolbilin and Shtogrin [7] showed for $n=3$ and $k=1$ that the condition $\rho>10 R$ suffices. Their proof methods do not apply in dimensions $n \geq 4$. Engel [9, Theorem 9.9] announced a general result of this type for $k=1$, namely that (1.4) can then be weakened to the condition $\rho>6 R$, independent of the dimension. However his proof is incomplete, since it depends on his unproved Conjecture 9.7.

In this paper $\log x$ and $\log _{2} x$ represent logarithms to base $e$ and 2, respectively.

\section{Crystallographic Groups}

In this section we give several characterizations of crystallographic groups, most of which are well known. First, we set some notation. The Euclidean norm on $\mathbb{R}^{n}$ is denoted $\|\mathbf{x}\|=\left(\sum_{i=1}^{n} x_{i}^{2}\right)^{1 / 2}$. For $\rho>0$, let $B(\mathbf{x} ; \rho)$ denote the closed ball of radius $\rho$ around $\mathbf{x}$, given by

$$
B(\mathbf{x} ; \rho):=\{\mathbf{y}:\|\mathbf{y}-\mathbf{x}\| \leq \rho\} .
$$

Let $B^{\circ}(\mathbf{x} ; \rho)$ denote the corresponding open ball and $\partial B(\mathbf{x} ; \rho)$ its boundary,

$$
\partial B(\mathbf{x} ; \rho):=\{\mathbf{y}:\|\mathbf{y}-\mathbf{x}\|=\rho\} .
$$

A subset $X$ of $\mathbb{R}^{n}$ is discrete if it is closed and for each $\mathbf{x} \in X$ there is a radius $\rho_{\mathbf{x}}>0$ such that

$$
X \cap B\left(\mathbf{x} ; \rho_{\mathbf{x}}\right)=\{\mathbf{x}\} .
$$

Let $\mathbb{E}^{n}$ denote the space $\mathbb{R}^{n}$ endowed with the Euclidean metric. The Euclidean group $\mathcal{E}_{n}=\operatorname{Sym}\left(\mathbb{E}^{n}\right)$ denotes the group of isometries of $\mathbb{R}^{n}$. It is a real Lie group of dimension $n(n+1) / 2$ and is a semidirect product of the orthogonal group $O(n, \mathbb{R})=$ 
$\left\{Q: Q Q^{T}=I\right\}$ and the translation group $\mathbb{R}^{n}$. We denote its elements $g=(Q, \mathbf{t})$ where $Q$ is the rotational part of $g$ and $\mathbf{t}$ is the translational part of $g$, with multiplication

$$
g^{\prime} g=\left(Q^{\prime}, \mathbf{t}^{\prime}\right)(Q, \mathbf{t})=\left(Q^{\prime} Q, \mathbf{t}^{\prime}+Q^{\prime} \mathbf{t}\right)
$$

A general element $g \in \mathcal{E}_{n}$ acts on $\mathbb{R}^{n}$ by

$$
g \cdot \mathbf{x}=Q \mathbf{x}+\mathbf{t}
$$

The following definitions concern subgroups $G$ of the Euclidean group $\mathcal{E}^{n}$.

Definition 2.1. A group $G$ is a discrete subgroup of $\mathcal{E}_{n}$ if it is a closed subgroup of $\mathcal{E}_{n}$ and the topology induced on $G$ from the standard (Euclidean) topology on $\mathcal{E}_{n}$ is discrete. That is, for each $g \in G$ there is an open set $U$ of $\mathcal{E}_{n}$ such that $G \cap U=\{g\}$.

Definition 2.2. A group $G$ acts discontinuously ${ }^{1}$ on $\mathbb{R}^{n}$ if for every $\mathbf{x} \in \mathbb{R}^{n}$ the orbit

$$
G \cdot \mathbf{x}:=\{g \cdot \mathbf{x}: g \in G\}
$$

is a discrete set in $\mathbb{R}^{n}$.

In this definition we allow the possibility that there are distinct $g_{1}, g_{2} \in G$ with $g_{1} \cdot \mathbf{x}=g_{2} \cdot \mathbf{x}$.

Definition 2.3. A group $G$ acts uniformly on $\mathbb{R}^{n}$ if the orbit space $\mathbb{R}^{n} / G$ is compact in the quotient topology.

This definition implies that the translational parts $\mathbf{t}$ of elements of $G$ span $\mathbb{R}^{n}$ as a vector space. Indeed, call a group $G$ of isometries irreducible if for each invertible affine map $\alpha: \mathbb{R}^{n} \rightarrow \mathbb{R}^{n}$ the group $G^{\prime}=\alpha G \alpha^{-1}$ has the property that the translational parts of all elements of $G^{\prime}$ span $\mathbb{R}^{n}$. A group $G$ of isometries is irreducible if and only if it acts uniformly on $\mathbb{R}^{n}$, see Charlap [3, p. 3].

Definition 2.4. A group $G$ is crystallographic if it acts uniformly and discontinuously on $\mathbb{R}^{n}$. (This is the definition of Charlap [3, p. 4].)

Definition 2.5. A group $G$ is a space group if the subgroup $T$ of all pure translations in $G$ is an $n$-dimensional lattice. (See Schwarzenberger [23, p. 26].)

The following theorem gives several necessary and sufficient conditions for a group $G$ to be crystallographic. All of these criteria are quite well known, except for (v), which will be the criterion we mainly use in this paper.

\footnotetext{
${ }^{1}$ Schwarzenberger [23] terms this "acts discretely on $\mathbb{R}^{n}$. "
} 
Theorem 2.1. The following properties of a group $G$ contained in the Euclidean group $\mathcal{E}_{n}=\operatorname{Sym}\left(\mathbb{E}^{n}\right)$ are equivalent:

(i) $G$ is a crystallographic group. That is, $G$ acts uniformly and discontinuously on $\mathbb{R}^{n}$.

(ii) $G$ is a space group. That is, the subgroup of pure translations $T$ in $G$ is an $n$-dimensional lattice, which is necessarily the maximal abelian subgroup of $G$.

(iii) $G$ is a discrete subgroup of $\mathcal{E}_{n}$ and $\mathcal{E}_{n} / G$ is compact.

(iv) Every orbit $G \cdot \mathbf{x}=\{g \cdot \mathbf{x}: g \in G\}$ is a Delone set in $\mathbb{R}^{n}$.

(v) There exists one orbit $G \cdot \mathbf{x}$ which is a discrete, relatively dense set in $\mathbb{R}^{n}$.

Remark. We later obtain an even weaker version of (v) in Corollary 3.1.

Proof. (i) $\Leftrightarrow$ (ii) The direction (i) $\Rightarrow$ (ii) is Bieberbach's first theorem, see [2], [3, p. 17], [18], or [31]. Besides being of finite index in $G, T$ is the unique maximal, normal, abelian subgroup of $G$, see [3, p. 18]. For (ii) $\Rightarrow$ (i) we use [3, p. 4 bottom] to obtain that $G$ acts uniformly. Next $T$ is of finite index in $G$ [23, p. 26] and acts discontinuously on $\mathbb{R}^{n}$. We complete this step by using the observation that if $G$ contains a subgroup of finite index that acts discontinuously on $\mathbb{R}^{n}$, then $G$ also acts discontinuously on $\mathbb{R}^{n}$.

(ii) $\Rightarrow$ (iii) This is a theorem in [23, p. 27].

(iii) $\Rightarrow$ (i) This is also a theorem of Bieberbach, see [23, p. 29].

(ii) $\Rightarrow$ (iv) Any orbit $T \cdot \mathbf{x}$ is a translate of the $n$-dimensional lattice $T \cdot \mathbf{0}$. Since [ $G: T]$ is finite and $T$ is a normal subgroup of $G$, the set $G \cdot \mathbf{x}$ consists of $[G: T]$ translates of $T \cdot \mathbf{0}$, hence it is a Delone set.

(iv) $\Rightarrow$ (v) Trivial.

(v) $\Rightarrow$ (iii) Since $G \cdot \mathbf{x}$ is relatively dense, the vectors in $G \cdot \mathbf{x}$ span $\mathbb{R}^{n}$, because they clearly cannot all lie in an $(n-1)$-dimensional affine subspace. This, together with $G \cdot \mathbf{x}$ being a discrete set, implies that any element $g$ of $G$ sufficiently close to the identity $e$ must be the identity, hence $G$ is a discrete subgroup of $\mathcal{E}_{n}$.

To show that $\mathcal{E}_{n} / G$ is compact, it suffices to show that for any $g^{\prime} \in \mathcal{E}_{n}$ with $g^{\prime} \cdot \mathbf{z}=$ $Q^{\prime} \mathbf{z}+\mathbf{t}^{\prime}$ we can find some element $g \in G$, say $g \cdot \mathbf{z}=Q \mathbf{z}+\mathbf{t}$, such that the element $g^{\prime} g$ with

$$
g^{\prime} g \cdot \mathbf{z}=Q^{\prime} Q \mathbf{z}+Q^{\prime} \mathbf{t}+\mathbf{t}^{\prime}
$$

has $\left\|Q^{\prime} \mathbf{t}+\mathbf{t}^{\prime}\right\|$ bounded, because any region $O(n, \mathbb{R}) \times\left\{\mathbf{t}:\|\mathbf{t}\| \leq R^{\prime}\right\}$, for any $R^{\prime}$, is a compact subset of $\mathcal{E}_{n}$. Let $\|Q\|_{2}$ denote the Frobenius norm on matrices, which is

$$
\|Q\|_{2}=\left(\sum_{i, j=1}^{n} Q_{i j}^{2}\right)^{1 / 2}=\left(\operatorname{Tr}\left(Q Q^{T}\right)\right)^{1 / 2},
$$

and note that orthogonal matrices $Q$ satisfy $\|Q\|_{2}=\sqrt{n}$. We now view the orbit $G \cdot \mathbf{x}$ as generated by that element $\mathbf{x}$ in the orbit which minimizes $\|\mathbf{x}\|$. Then $\|\mathbf{x}\| \leq R$ since $G \cdot \mathbf{x}$ is relatively dense with constant $R$. By relative denseness we can also find $\mathbf{y} \in G \cdot \mathbf{x}$ such that

$$
\left\|\mathbf{y}-\left(Q^{\prime}\right)^{-1} \mathbf{t}^{\prime}\right\| \leq R
$$


Next choose a $g \in G$ with $\mathbf{y}=g \cdot \mathbf{x}=Q \mathbf{x}+\mathbf{t}$. Then $g^{\prime} g$ has translation part satisfying

$$
\begin{aligned}
\left\|Q^{\prime} \mathbf{t}+\mathbf{t}^{\prime}\right\| & =\left\|Q^{\prime}(\mathbf{y}-Q \mathbf{x})+\mathbf{t}^{\prime}\right\| \\
& \leq\left\|Q^{\prime}\right\|_{2}\left\|\mathbf{y}-\left(Q^{\prime}\right)^{-1} \mathbf{t}^{\prime}\right\|+\left\|Q^{\prime}\right\|_{2}\|Q\|_{2}\|\mathbf{x}\| \\
& \leq(\sqrt{n}+n) R,
\end{aligned}
$$

which is the desired bound.

From Theorem 2.1 we easily deduce Theorem 1.1 .

Proof of Theorem 1.1. (i) $\Rightarrow$ (iii) Choose a radius $\rho$ sufficiently large that the ball $B(\mathbf{0} ; \rho)$ contains representatives $\left\{\mathbf{x}_{i}: 1 \leq i \leq|\mathrm{ST}(X)|\right\}$ of each isometry class of global star $\mathrm{ST}_{\mathbf{x}_{i}}(X)$. For any $\mathbf{x} \in X$ there exists some $\mathbf{x}_{j}$ such that $\mathrm{ST}_{\mathbf{x}}(X)$ is isometric to $\operatorname{ST}_{\mathbf{x}_{j}}(X)$ via an isometry $g=g\left(\mathbf{x}, \mathbf{x}_{j}\right) \in \operatorname{Sym}(X)$. Then for each $i$,

$$
\left\|g^{-1} \cdot \mathbf{x}_{i}-\mathbf{x}\right\|=\left\|g^{-1} \cdot\left(\mathbf{x}_{i}-\mathbf{x}_{j}\right)\right\| \leq 2 \rho
$$

and $\mathrm{ST}_{g^{-1} \cdot \mathbf{x}_{i}}(X)$ is isometric to $\operatorname{ST}_{\mathbf{x}_{i}}(X)$. Thus each of the sets

$$
X_{i}:=\left\{\mathbf{x} \in X: \operatorname{ST}_{\mathbf{x}}(X) \quad \text { is isometric to } \operatorname{ST}_{\mathbf{x}_{i}}(X)\right\}
$$

is relatively dense with constant $2 \rho+R$. Since

$$
X_{i} \subseteq \operatorname{Sym}(X) \cdot \mathbf{x}_{i} \subseteq X,
$$

it follows that the $\operatorname{Sym}(X)$-orbit of $\mathbf{x}_{i}$ is discrete and relatively dense, hence $\operatorname{Sym}(X)$ is a crystallographic group by property (v) of Theorem 2.1 .

(iii) $\Rightarrow$ (ii) $X$ is invariant under the maximal abelian subgroup $T$ contained in $\operatorname{Sym}(X)$, which is an $n$-dimensional lattice since $\operatorname{Sym}(X)$ is a crystallographic group. Now $X$ is a union of orbits $T \cdot \mathbf{x}$, each of which is a translate of the lattice $L=T \cdot \mathbf{0}$. Since $X$ is a discrete set, there can only be finitely many such orbits.

(ii) $\Rightarrow$ (i) Immediate.

For each crystallographic group $G$ there exists some multiregular point system such that $G=\operatorname{Sym}(X)$. This contrasts with the situation for regular point systems. For example, there is no regular point system $X$ with $\operatorname{Sym}(X)$ equal to a group $G$ of pure translations $\mathbb{Z}^{n}$. Indeed, the orbit $G \cdot \mathbf{x}$, which is a translate of a full-rank lattice, has a strictly larger symmetry group than $G$, for $\operatorname{Sym}(G \cdot \mathbf{x})$ contains a reflection $g \cdot \mathbf{y}:=\mathbf{x}_{0}-\mathbf{y}$ around any given point $\mathbf{x}_{0} \in G \cdot \mathbf{x}$. For this reason the classification of regular point systems differs from that of crystallographic groups.

We conclude this section with the following consequence of Theorem 1.1. Call a set $X$ in $\mathbb{R}^{n}$ fully periodic if it is invariant under some full rank lattice $L$ of translations. We have: If $X$ is a Delone set, then $X$ is not fully periodic if and only if $|\mathrm{ST}(X)|$ is infinite.

\section{Global Star Criterion}

We show that a discrete set $X$ is an ideal crystal provided that certain sets of global stars $\left\{\mathrm{St}_{\mathbf{y}}(X): \mathbf{y} \in Y\right\}$ are all isometric. 
Definition 3.1. An arbitrary set $Y$ in $\mathbb{R}^{n}$ is an enclosing set if there is a point $\mathbf{y} \in Y$ which is contained in the interior of the convex hull of the remaining points $Y \backslash\{\mathbf{y}\}$. We call any such $\mathbf{y} \in Y$ an enclosed point.

This definition requires that the convex hull of $Y$ in $\mathbb{R}^{n}$ be full dimensional, i.e., the points of $Y$ do not all lie in some hyperplane. An enclosing set $Y$ necessarily has cardinality $|Y| \geq n+2$, and $|Y|=n+2$ occurs when $Y$ consists of the vertices of a $n$-simplex plus one point in the interior of the simplex. We prove:

Theorem 3.1. Let $X$ be a discrete set in $\mathbb{R}^{n}$ and suppose that $Y$ is a (finite or infinite) set such that all the stars $\left\{\mathrm{ST}_{\mathbf{y}}(X): \mathbf{y} \in Y\right\}$ are isometric. If $Y$ is an enclosing set, then $\operatorname{Sym}(X)$ is a crystallographic group, and $X$ is an ideal crystal.

In this result the set $X$ and the star centers $Y$ are not necessarily related, and we may have $X \cap Y=\emptyset$. Theorem 1.2 directly follows as a special case of Theorem 3.1.

Theorem 3.1 also gives the following relaxation of property (v) in Theorem 2.2.

Corollary 3.1. If $G$ is a group of isometries of $\mathbb{R}^{n}$ that contains an orbit $G \cdot \mathbf{x}$ which is discrete and is also an enclosing set, then $G$ is a crystallographic group.

Proof. Apply Theorem 3.1 with $Y=X=G \cdot \mathbf{x}$.

Before proving Theorem 3.1, we derive some equivalent conditions for a set $Y$ to be an enclosing set.

Definition 3.2. Given $\rho>0$, a point $\mathbf{y}$ of a set $Y$ in $\mathbb{R}^{n}$ is a $\rho$-point of $Y$ if every ball $B(\mathbf{x} ; \rho)$ that contains $\mathbf{y}$ on its boundary $\partial B(\mathbf{x} ; \rho)$ also contains a point of $Y \backslash\{\mathbf{y}\}$.

Definition 3.3. An oriented hyperplane $H$ is a hyperplane

$$
H:=H_{\mathbf{c}, d}=\{\mathbf{x}:\langle\mathbf{c}, \mathbf{x}\rangle=d\}
$$

together with a particular labeling $\mathrm{H}^{+}, \mathrm{H}^{-}$of the two open half-spaces it determines. The data $(\mathbf{c}, d)$ with $\mathbf{x} \neq \mathbf{0}$ determine these open half-spaces by

$$
H^{+}=\{\mathbf{x}:\langle\mathbf{c}, \mathbf{x}\rangle>d\} \quad \text { and } \quad H^{-}=\{\mathbf{x}:\langle\mathbf{c}, \mathbf{x}\rangle<d\} .
$$

We prove:

Lemma 3.1. The following conditions on a point $\mathbf{y}$ in a set $Y$ in $\mathbb{R}^{n}$ are equivalent.

(i) $\mathbf{y}$ is an enclosed point of $Y$, i.e., $\mathbf{y}$ is in the interior of the convex hull of $Y \backslash\{\mathbf{y}\}$.

(ii) $\mathbf{y}$ is a $\rho$-point of $Y$, for some finite $\rho>0$.

(iii) Every oriented hyperplane $H$ containing $\mathbf{y}$ satisfies

$$
H^{+} \cap Y \neq \emptyset \quad \text { and } \quad H^{-} \cap Y \neq \emptyset .
$$


Proof. (i) $\Rightarrow$ (iii) We prove the contrapositive. Suppose (iii) fails. If some hyperplane $H$ has $\operatorname{Int}\left(H^{+} \cap Y\right)=\emptyset$, then the convex hull of $Y$ is contained in the closed half-space $\bar{H}^{-}=H \cup H^{-}$, and $\mathbf{y} \in H$ is on its boundary, hence $\mathbf{y}$ cannot be an (n-dimensional) interior point of the convex hull of $Y$, and (i) fails.

(iii) $\Rightarrow$ (ii) We prove the contrapositive. Suppose (ii) fails. Then there are arbitrarily large closed balls $B\left(\mathbf{x}_{j} ; \rho_{j}\right)$ which have $\mathbf{y}$ on their boundary and contain other no point of $Y$. Let $H_{j}$ be the tangent hyperplane to $\partial B\left(\mathbf{x}_{j} ; \rho_{j}\right)$ at $\mathbf{y}$, oriented so that $B\left(\mathbf{x}_{j} ; \rho_{j}\right) \backslash\{\mathbf{y}\} \subseteq$ $H^{+}$. Extract a subsequence of $H_{j}$ that tends to a limit hyperplane $H$ containing $\mathbf{y}$. Along this subsequence the sets $B\left(\mathbf{x}_{j} ; \rho_{j}\right) \backslash\{\mathbf{y}\}$ exhaust all of $H^{+}$, hence $H^{+}$contains no point of $Y$, contradicting (iii).

(ii) $\Rightarrow$ (i) Let $\tilde{Y}(\mathbf{y}):=Y \cap B(\mathbf{y} ; 2 \rho)$, which consists of all points of $Y$ contained in some closed ball $B(\mathbf{x} ; \rho)$ that contains $\mathbf{y}$ on its boundary. We show that $\tilde{Y}$ contains $\mathbf{y}$ as an enclosed point. Suppose not. Then there is an oriented hyperplane $H$ through $\mathbf{y}$ which contains no point of $Y \backslash\{\mathbf{y}\}$ on one side $H^{+}$of it. Consider the closed ball $B(\mathbf{x} ; \rho)$ contained in $H^{+}$which is tangent to $H$ at $\mathbf{y}$. It contains no point of $Y \backslash\{\mathbf{y}\}$, which contradicts (ii).

We also need the following simple criterion for a set $Y$ to be relatively dense.

Lemma 3.2. An arbitrary closed set $Y$ in $\mathbb{R}^{n}$ is relatively dense if and only if there is some $\rho>0$ such that every point $\mathbf{y} \in Y$ is a $\rho$-point of $Y$.

Proof. If $Y$ is relatively dense with parameter $R$, then every point $\mathbf{y} \in Y$ is an $R$-point. Indeed, relative denseness implies that every ball $B(\mathbf{x}, R)$ with $\mathbf{y} \in \partial B(\mathbf{x}, R)$ contains some other point of $Y$.

Conversely, if every point of $Y$ is a $\rho$-point, for fixed $\rho>0$, then $Y$ is relatively dense with parameter $\rho$. Suppose not, so that there is some empty ball $B\left(\mathbf{z} ; \rho^{\prime}\right)$ with $\rho^{\prime}>\rho$. Choose a point $\mathbf{y} \in Y$ that is a closest point to $\mathbf{z}$, and translate the ball in the direction $\mathbf{y}-\mathbf{z}$ until $\mathbf{y} \in Y$ is on its boundary. The resulting ball $B\left(\mathbf{z}^{\prime} ; \rho\right)$ contains no point of $Y \backslash\{\mathbf{y}\}$ by the triangle inequality, for any other point in it would be closer to $\mathbf{z}$ than $\mathbf{y}$. This contradicts $\mathbf{y}$ being a $\rho$-point of $Y$.

Proof of Theorem 3.1. By hypothesis, for each point $\mathbf{y}_{i}, \mathbf{y}_{j}$ of $Y$ there is an isometry $g_{i j}$ of $\mathbb{R}^{n}$ that maps $\mathbf{y}_{i}$ to $\mathbf{y}_{j}$ and $\operatorname{ST}_{\mathbf{y}_{i}}(X)$ to $\mathrm{ST}_{\mathbf{y}_{j}}(X)$. We do not require that any of the $\mathbf{y}_{i}$ be in $X$. Let $G$ be the group of isometries generated by all the possible $g_{i j}$ 's. Then $Y \subseteq G \cdot \mathbf{y}$ for any fixed $\mathbf{y} \in Y$. Since each $g_{i j} \in \operatorname{Sym}(X)$, we have $G \subseteq \operatorname{Sym}(X)$. The main part of the proof will be to show that $G$ is a crystallographic group.

Consider an orbit $G \cdot \mathbf{x}$ for some $\mathbf{x} \in X$ and let $\operatorname{Aff}(G \cdot \mathbf{x})$ denote the smallest affine subspace spanned by the elements of $G \cdot \mathbf{x}$. We show that $\operatorname{dim}(\operatorname{Aff}(X))=n$. Assume the contrary, that $G \cdot \mathbf{x}$ spans some affine subspace $P^{k}=\operatorname{Aff}(G \cdot \mathbf{x})$ of dimension $k<n$. Now $G$ must leave $P^{k}$ invariant, i.e., $g\left(P^{k}\right)=P^{k}$ for all $g \in G$. Since $g\left(P^{k}\right)=P^{k}$ for all $g \in G$, we have

$$
\operatorname{dist}\left(\mathbf{y}, P^{k}\right)=\operatorname{dist}\left(g \cdot \mathbf{y}, P^{k}\right),
$$


where $\operatorname{dist}(\mathbf{y}, F):=\inf \{\|\mathbf{y}-\mathbf{z}\|: \mathbf{z} \in F\}$. Thus the orbit $G \cdot \mathbf{y}$ lies on a cylinder

$$
C_{\alpha}:=\left\{\mathbf{z} \in \mathbb{R}^{n}: \operatorname{dist}\left(\mathbf{z}, P^{k}\right)=\alpha\right\} .
$$

The constant $\alpha>0$, for if $\alpha=0$, then $Y \subseteq G \cdot \mathbf{y} \subseteq P^{k}$, which contradicts the property that the points of an enclosing set $Y$ span $\mathbb{R}^{n}$. If $k<n$, then the cylinder $C_{\alpha} \cong P^{k} \times S^{n-k}$ is a convex set, hence a supporting hyperplane $H$ to a point $\mathbf{y} \in Y$ on the surface of the cylinder leaves the entire cylinder $C_{\alpha}$ in one closed half-space $\bar{H}=H \cup H^{-}$, say. Then $H^{+} \cap Y=\emptyset$, which by Lemma 3.1(iii) shows that $Y$ is not an enclosing set, a contradiction. Thus $k=n$.

We next show that $\operatorname{Sym}(X)$ acts discontinuously on $\mathbb{R}^{n}$. We must show that every orbit $\operatorname{Sym}(X) \cdot \mathbf{z}$ is a discrete set. We know that $\operatorname{Sym}(X) \cdot \mathbf{x} \subseteq X$ is a discrete set, and $\operatorname{Aff}(\operatorname{Sym}(X) \cdot \mathbf{x})=\mathbb{R}^{n}$ because $\mathbb{R}^{n}=\operatorname{Aff}(G \cdot \mathbf{x}) \subseteq \operatorname{Aff}(\operatorname{Sym}(X) \cdot \mathbf{x})$. Now

$$
\operatorname{Aff}(\operatorname{Sym}(X) \cdot \mathbf{x})=\mathbb{R}^{n}
$$

implies that there exist $n+1$ points $\left\{\mathbf{x}_{i}: 1 \leq i \leq n+1\right\}$ which form a full-dimensional simplex in $\mathbb{R}^{n}$, so we can write

$$
\mathbf{z}=\sum_{i=1}^{n+1} a_{i} \mathbf{x}_{i} \quad \text { with } \quad \sum_{i=1}^{n+1} a_{i}=1 .
$$

Here $\left(a_{1}, a_{2}, \ldots, a_{n+1}\right)$ are barycentric coordinates of $\mathbf{z}$ and

$$
\left\|\mathbf{z}-\mathbf{x}_{i}\right\| \leq c_{0}:=\sum_{i=1}^{n+1}\left(\left|a_{i}\right|+1\right)\left\|\mathbf{x}_{i}\right\|, \quad 1 \leq i \leq n+1 .
$$

We now argue by contradiction. Suppose that $\operatorname{Sym}(X) \cdot \mathbf{z}$ is not discrete. Then there exists a sequence of distinct points $\left\{\mathbf{z}_{j}=g_{j} \cdot \mathbf{z}: j \geq 1\right\}$ tending to a limit point $\mathbf{z}_{\infty}$. However

$$
g_{j} \cdot \mathbf{z}=\sum_{i=1}^{n+1} a_{i}\left(g_{j} \cdot \mathbf{x}_{i}\right),
$$

and all $g_{j} \cdot \mathbf{x}_{i} \in \operatorname{Sym}(X) \cdot \mathbf{x}$. Now we have

$$
\left\|\mathbf{z}_{\infty}-g_{j} \cdot \mathbf{x}_{i}\right\| \leq\left\|\mathbf{z}_{\infty}-\mathbf{z}_{j}\right\|+\left\|g_{j} \cdot \mathbf{z}-g_{j} \cdot \mathbf{x}_{i}\right\| \leq\left\|\mathbf{z}_{\infty}-\mathbf{z}_{j}\right\|+c_{0} .
$$

Whenever $\left\|\mathbf{z}_{\infty}-\mathbf{z}_{j}\right\| \leq 1$, the point $g_{j} \cdot \mathbf{x}_{i}$ lies in the fixed ball $B\left(\mathbf{z}_{\infty} ; c_{0}+1\right)$. Since $X$ is discrete, there are only finitely points of $\operatorname{Sym}(X) \cdot \mathbf{x}$ in this ball, hence by (3.1) there are only finitely many possible choices for $g_{j} \cdot \mathbf{z}$ having $\left\|\mathbf{z}_{\infty}-g_{j} \cdot \mathbf{z}\right\| \leq 1$, which contradicts the distinctness of all $\mathbf{z}_{j}$. Thus $\operatorname{Sym}(X)$ acts discontinuously on $\mathbb{R}^{n}$. In particular, $\operatorname{Sym}(X) \cdot \mathbf{y}$ is a discrete set, hence $G \cdot \mathbf{y}$ is a discrete set.

Now choose $\mathbf{y}$ to be an enclosed point of $Y$, so it is a $\rho$-point for some $\rho>0$ by Lemma 3.1. Now for any isometry $g \in G$, the point $g \cdot \mathbf{y}$ is a $\rho$-point of $g(Y)$, because property (iii) of Lemma 3.1 is invariant under isometries. Since $Y \subseteq G \cdot \mathbf{y}$ we have $g(Y) \subseteq G \cdot \mathbf{y}$, hence $g \cdot \mathbf{y}$ is a $\rho$-point of $Y$. Thus every point of $G \cdot \mathbf{y}$ is a $\rho$-point, so $G \cdot \mathbf{y}$ is a relatively dense set by Lemma 3.2. 
It follows that $G$ is a crystallographic group by property (v) of Theorem 2.1 . Thus $\mathbb{R}^{n} / G$ is compact, hence $\mathbb{R}^{n} / \operatorname{Sym}(X)$ is also compact, so $\operatorname{Sym}(X)$ acts uniformly on $\mathbb{R}^{n}$. We conclude that $\operatorname{Sym}(X)$ is a crystallographic group. Theorem 2.2 then shows that $X$ is an ideal crystal.

For later use we show that for any finite partition $X=\bigcup_{i=1}^{k} X_{i}$ of a relatively dense set, at least one set $X_{i}$ is an enclosing set. The following lemma shows that this conclusion holds more generally for finite sets $X$ that are relatively dense on any sufficiently large finite region. We say that a set $X$ is $R$-relatively dense in the ball $B(\mathbf{y} ; \rho)$ if every closed ball $B(\mathbf{z} ; R)$ contained in $B(\mathbf{y} ; \rho)$ contains a point of $X$.

Lemma 3.3. Let $X$ be a set in $\mathbb{R}^{n}$ which is partitioned into $k$ subsets,

$$
X=\bigcup_{i=1}^{k} X_{i} \quad \text { with } \quad X_{i} \cap X_{j}=\emptyset \quad \text { if } \quad i \neq j .
$$

If $X$ is $R$-relatively dense in some ball $B(\mathbf{y} ; \rho)$ with $\rho \geq 3^{k} R$, then at least one subset $X_{i}$ is an enclosing set.

Proof. We proceed by induction on $k$. For the base case $k=1$, if $X=X_{1}$ is $R$ relatively dense in $B(\mathbf{y} ; 3 R)$, then there exists some $\mathbf{x} \in X$ with $\|\mathbf{x}-\mathbf{y}\| \leq R$. The set $\tilde{X}(\mathbf{x})=X \cap B(\mathbf{x} ; 2 R)$ of all points of $X$ within distance $2 R$ of $\mathbf{x}$ contains $\mathbf{x}$ as an enclosed point, as in the proof of Lemma 3.1(ii) $\Rightarrow$ (i), because it includes each point of $X$ in any $B(\mathbf{z} ; R)$ that contains $\mathbf{x}$ on its boundary, and all such $B(\mathbf{z} ; R) \subseteq B(\mathbf{y} ; 3 R)$.

Now suppose that the lemma is true for all partitions of $X$ into $k-1$ subsets. Consider a partition of $X$ into $k$ subsets, and suppose that there is a ball $B\left(\mathbf{y} ; 3^{k} R\right)$ on which $X$ is $R$-relatively dense. If the set $X_{k}$ is $3^{k-1} R$-relatively dense on $B=B\left(\mathbf{y} ; 3^{k} R\right)$, then the set $X_{k}$ is an enclosing set, by applying the base case $k=1$. If it is not, then there is some ball $B^{\prime}=B\left(\mathbf{y}^{\prime} ; 3^{k-1} R\right)$ inside $B\left(\mathbf{y} ; 3^{k} R\right)$ that contains no point of $X_{k}$. Now set $X^{\prime}=X \cap B^{\prime}$ and $X_{i}^{\prime}=X_{i} \cap B^{\prime}, 1 \leq i \leq k-1$. Then since $X_{k}^{\prime}=X_{k} \cap B^{\prime}=\emptyset$, we have a partition

$$
X^{\prime}=\bigcup_{i=1}^{k-1} X_{i}^{\prime},
$$

and $X^{\prime}$ inherits the $R$-relatively dense property on $B\left(\mathbf{y}^{\prime} ; 3^{k-1} R\right)$ from that of $X$ on $B$. By the induction hypothesis one of the $X_{i}^{\prime}$ is an enclosing set, and since $X_{i}^{\prime} \subseteq X_{i}$ the enclosing set property holds for the corresponding $X_{i}$. This completes the induction step.

\section{Local Star Criterion}

We deduce results on the multiregularity of a point set $X$ from conditions on a set of its local stars ( $\rho$-stars) centered at a Delone set of points $Y$ contained in $X$. In Corollary 4.1 below we recover the main result stated in [8], which generalizes that of Delone et al. [6]. 
Recall the definition

$$
\operatorname{ST}_{\mathbf{y}}(X ; \rho):=\{[\mathbf{y}, \mathbf{x}]: \mathbf{x} \in X \text { and }\|\mathbf{x}-\mathbf{y}\| \leq \rho\} .
$$

This implies that

$$
\operatorname{ST}_{\mathbf{y}}(X ; \rho) \subseteq \operatorname{ST}_{\mathbf{y}}\left(X ; \rho^{\prime}\right) \quad \text { if } \quad \rho \leq \rho^{\prime}
$$

Definition 4.1. The local stabilizer of the $\rho$-star centered at $\mathbf{y}$, denoted $\operatorname{Lsym}_{\mathbf{y}}(X ; \rho)$, is the group of isometries $g$ that fix $\mathbf{y}$ and leave $\operatorname{ST}_{\mathbf{y}}(X ; \rho)$ invariant. That is, it consists of all $g \in \operatorname{Sym}\left(\mathbb{E}^{n}\right)$ such that $g \cdot \mathbf{y}=\mathbf{y}$ and

$$
g\left(\operatorname{ST}_{\mathbf{y}}(X ; \rho)\right)=\operatorname{ST}_{\mathbf{y}}(X ; \rho) .
$$

$\operatorname{Lsym}_{\mathbf{y}}(X ; \rho)$ is a subgroup of the group of rotations centered at $\mathbf{y}$. The relation (4.1) implies that

$$
\operatorname{Lsym}_{\mathbf{y}}(X ; \rho) \supseteq \operatorname{Lsym}_{\mathbf{y}}\left(X ; \rho^{\prime}\right) \quad \text { if } \quad \rho \leq \rho^{\prime} .
$$

Definition 4.2. The (global) stabilizer $\operatorname{Lsym}_{\mathbf{y}}(X)$ centered at $\mathbf{y}$ is the subgroup of elements of $\operatorname{Sym}(X)$ that fix $\mathbf{y}$.

We clearly have

$$
\operatorname{Lsym}_{\mathbf{y}}(X) \subseteq \operatorname{Lsym}_{\mathbf{y}}(X ; \rho) \quad \text { for any } \rho .
$$

Any group $\operatorname{Lsym}_{\mathbf{y}}(X ; \rho)$ or $\operatorname{Lsym}_{\mathbf{y}}(X)$ is conjugate in $\operatorname{Sym}\left(\mathbb{E}^{n}\right)$ to a subgroup of the orthogonal group $O(n, \mathbb{R})$ by applying the conjugacy that translates $\mathbf{y}$ to the origin, i.e., $t_{-\mathbf{y}} g t_{\mathbf{y}} \in O(n, \mathbb{R})$, where $t_{\mathbf{y}} \cdot \mathbf{x}=\mathbf{x}+\mathbf{y}$.

We generalize these definitions to apply to a partition $X=Y \cup Z$ of a set $X$. We define the partitioned $\rho$-star

$$
\operatorname{ST}_{\mathbf{w}}(Y, Z ; \rho):=\operatorname{ST}_{\mathbf{w}}(Y ; \rho) \cup \operatorname{ST}_{\mathbf{w}}(Z ; \rho)=\operatorname{ST}_{\mathbf{w}}(X ; \rho),
$$

and we consider two such partitioned $\rho$-stars isometric if there is an isometry $g \in \operatorname{Sym}\left(\mathbb{E}^{n}\right)$ with $g \cdot \mathbf{w}=\mathbf{w}^{\prime}$ which satisfies

$$
g\left(\operatorname{ST}_{\mathbf{w}}(Y ; \rho)\right)=\operatorname{ST}_{\mathbf{w}^{\prime}}(Y ; \rho) \quad \text { and } \quad g\left(\operatorname{ST}_{\mathbf{w}}(Z ; \rho)\right)=\operatorname{ST}_{\mathbf{w}^{\prime}}(Z ; \rho) .
$$

We call any such isometry $g$ a $(Y, Z ; \rho)$-isometry. We set

$$
\operatorname{ST}_{W}(Y, Z ; \rho):=\left\{\operatorname{ST}_{\mathbf{w}}(Y, Z ; \rho): \mathbf{w} \in W\right\},
$$

and let $\left|\operatorname{ST}_{W}(Y, Z ; \rho)\right|$ denote the number of $(Y, Z ; \rho)$-isometry classes among the members of $\operatorname{ST}_{W}(Y, Z ; \rho)$.

Finally, we define the local stabilizer $\operatorname{Lsym}_{\mathbf{w}}(Y, Z ; \rho)$ of the partitioned $\rho$-star by

$$
\operatorname{Lsym}_{\mathbf{w}}(Y, Z ; \rho)=\operatorname{Lsym}_{\mathbf{w}}(Y ; \rho) \cap \operatorname{Lsym}_{\mathbf{w}}(Z ; \rho),
$$

and the stabilizer $\operatorname{Lsym}_{\mathbf{w}}(Y, Z)$ by

$$
\operatorname{Lsym}_{\mathbf{w}}(Y, Z)=\operatorname{Lsym}_{\mathbf{w}}(Y) \cap \operatorname{Lsym}_{\mathbf{w}}(Z) .
$$


We obtain the following criterion:

Theorem 4.1. Let $X$ be a discrete set which has a partition $X=Y \cup Z$ in which $Y$ is a Delone set. Suppose that $\rho \geq 4 R$ is such that:

(a) $\left|\operatorname{ST}_{Y}(Y, Z ; \rho)\right|=\left|\operatorname{ST}_{Y}(Y, Z ; \rho-2 R)\right|=k$; and

(b) $\operatorname{Lsym}_{\mathbf{y}}(Y, Z ; \rho)=\operatorname{Lsym}_{\mathbf{y}}(Y, Z ; \rho-2 R)$ for all $\mathbf{y} \in Y$.

Then $\operatorname{Sym}(Y) \cap \operatorname{Sym}(Z) \subseteq \operatorname{Sym}(X)$ is a crystallographic group. Furthermore, each of $X$ and $Y$ is a multiregular point system, and the points of $X$ fall into at most $k$ orbits under the action of $\operatorname{Sym}(X)$.

In hypothesis (b) the groups $\operatorname{Lsym}_{\mathbf{y}}(Y, Z ; \rho)$ for $\mathbf{y} \in Y$ are not all necessarily isomorphic. The proof implies that they fall in at most $k$ isomorphism classes. Lemma 4.2 below shows that, for any value of $\rho$ that satisfies (a) and (b), we have

$$
\operatorname{Lsym}_{\mathbf{y}}(Y, Z)=\operatorname{Lsym}_{\mathbf{y}}(Y, Z ; \rho) \quad \text { all } \quad \mathbf{y} \in Y \text {. }
$$

Theorem 4.1 states nothing about the group $\operatorname{Sym}(Z)$, but the proof implies that $\operatorname{Sym}(Z)$ acts uniformly on $\mathbb{R}^{n}$. The theorem includes the special case $Z=\emptyset$, where we make the convention that $\operatorname{Sym}(\emptyset)=\operatorname{Sym}\left(\mathbb{E}^{n}\right)=\mathcal{E}_{n}$. This special case is:

Corollary 4.1. Let $X$ be an $(r, R)$ set for which there exists some $\rho \geq 4 R$ such that:

(a) $\left|\operatorname{ST}_{X}(X ; \rho)\right|=\left|\mathrm{ST}_{X}(X ; \rho-2 R)\right|=k$; and

(b) $\operatorname{Lsym}_{\mathbf{x}}(X ; \rho)=\operatorname{Lsym}_{\mathbf{x}}(X ; \rho-2 R)$ for all $\mathbf{x} \in X$.

Then $X$ is a multiregular point system whose points fall in exactly $k$ orbits under the action of $\operatorname{Sym}(X)$. Furthermore,

$$
\operatorname{Lsym}_{\mathbf{x}}(X)=\operatorname{Lsym}_{\mathbf{x}}(X ; \rho) \quad \text { for all } \mathbf{x} \in X .
$$

Here there are exactly $k$ orbits because condition (a) asserts that there are at least $k$ distinct orbits under the action of $\operatorname{Sym}(X)$.

An important part of the proof of Theorem 4.1 is to show that the set $X$ possesses global symmetries. We establish this in two preliminary lemmas, in which we assume all hypotheses of Theorem 4.1 are in force.

Lemma 4.1. Let $\mathbf{y}, \mathbf{y}^{\prime} \in Y$ and suppose $\rho \geq 4 R$ and that there exists $g \in \operatorname{Sym}\left(\mathbb{E}^{n}\right)$ which is a $(Y, Z ; \rho-2 R)$-isometry that maps $\operatorname{ST}_{\mathbf{y}}(X ; \rho-2 R)$ to $\operatorname{ST}_{\mathbf{y}^{\prime}}(X ; \rho-2 R)$. Then $g$ is also a $(Y, Z ; \rho)$-isometry that maps $\operatorname{ST}_{\mathbf{y}}(X ; \rho)$ to $\operatorname{ST}_{\mathbf{y}^{\prime}}(X ; \rho)$.

Proof. The hypothesis (a) in Theorem 4.1 implies that the isometry class of a star $\operatorname{ST}_{\mathbf{y}}(Y, Z ; \rho-2 R)$ uniquely determines the isometry class of the $\operatorname{star}_{\operatorname{ST}_{\mathbf{y}}}(Y, Z ; \rho)$. Thus the $(Y, Z ; \rho-2 R)$-isometry hypothesis implies that $\operatorname{ST}_{\mathbf{y}}(X ; \rho)$ is $(Y, Z ; \rho)$ isometric to $\operatorname{ST}_{\mathbf{y}^{\prime}}(X ; \rho)$, and we let $g^{\prime} \in \operatorname{Sym}\left(\mathbb{E}^{n}\right)$ be such an isometry between them. Now

$$
g^{-1} \circ g^{\prime}\left(\operatorname{ST}_{\mathbf{y}}(X ; \rho-2 R)\right)=g^{-1}\left(\operatorname{ST}_{\mathbf{y}^{\prime}}(X ; \rho-2 R)\right)=\operatorname{ST}_{\mathbf{y}}(X ; \rho-2 R) .
$$


Thus $g^{-1} \circ g^{\prime} \in \operatorname{Lsym}_{\mathbf{y}}(X ; \rho-2 R)$. Now hypothesis (b) of Theorem 4.1 gives

$$
g^{\prime \prime}=g^{-1} \circ g^{\prime} \in \operatorname{Lsym}_{\mathbf{y}}(X ; \rho) .
$$

Thus $g=g^{\prime} \circ\left(g^{\prime \prime}\right)^{-1}$ is a $(Y, Z ; \rho)$-isometry.

Next we show that any such local symmetry is a global symmetry of both $Y$ and $Z$.

Lemma 4.2. Let $\mathbf{y}, \mathbf{y}^{\prime} \in Y$ and suppose $\rho \geq 4 R$ and that there is a $(Y, Z ; \rho-2 R)$ isometry $g \in \operatorname{Sym}\left(\mathbb{E}^{n}\right)$ from $\mathbf{y}$ to $\mathbf{y}^{\prime}$, so that

$$
g\left(\operatorname{ST}_{\mathbf{y}}(X ; \rho-2 R)\right)=\operatorname{ST}_{\mathbf{y}^{\prime}}(X ; \rho-2 R) .
$$

Then $g \in \operatorname{Sym}(Y) \cap \operatorname{Sym}(Z)$. In particular,

$$
\operatorname{Lsym}_{\mathbf{y}}(Y, Z)=\operatorname{Lsym}_{\mathbf{y}}(Y, Z ; \rho) .
$$

Proof. Since $Y$ is an $(r, R)$-set, we can connect $\mathbf{y}$ to any $\overline{\mathbf{y}} \in Y$ by a finite chain $\mathbf{y}_{0}, \mathbf{y}_{1}, \ldots, \mathbf{y}_{m}$ with $\mathbf{y}_{0}=\mathbf{y}$ and $\mathbf{y}_{m}=\overline{\mathbf{y}}$, such that

$$
\left\|\mathbf{y}_{i}-\mathbf{y}_{i-1}\right\| \leq 2 R \quad \text { for } \quad 1 \leq i \leq m .
$$

This is proved in [6, Section 2], as follows. If $\|\overline{\mathbf{y}}-\mathbf{y}\|=R^{\prime}$, then the closed ball $\bar{B}=B\left(\overline{\mathbf{y}} ; R^{\prime}\right)$ contains only finitely many points of $Y$, and $\mathbf{y}$ lies on its boundary. The closed ball

$$
\bar{B}_{0}:=B\left(\mathbf{y}+\frac{R}{R^{\prime}}(\overline{\mathbf{y}}-\mathbf{y}) ; R\right) \subseteq \bar{B}
$$

contains $\mathbf{y}_{0}=\mathbf{y}$ on its boundary, and by relative denseness it must contain another point $\mathbf{y}_{1} \in Y$. Then $\left\|\mathbf{y}_{1}-\mathbf{y}_{0}\right\| \leq 2 R$ and

$$
\left\|\mathbf{y}_{1}-\overline{\mathbf{y}}\right\|<\left\|\mathbf{y}_{0}-\overline{\mathbf{y}}\right\|,
$$

since $\mathbf{y}$ is the furthest point in $\bar{B}_{0}$ from $\overline{\mathbf{y}}$. Now $\mathbf{y}_{1} \in \bar{B}$, so we can repeat the same argument to find $\mathbf{y}_{2} \in Y \cap \bar{B}$ with $\left\|\mathbf{y}_{2}-\mathbf{y}_{1}\right\| \leq 2 R$ and

$$
\left\|\mathbf{y}_{2}-\overline{\mathbf{y}}\right\|<\left\|\mathbf{y}_{1}-\overline{\mathbf{y}}\right\| .
$$

Continue this process to produce $\mathbf{y}_{3}, \mathbf{y}_{4}, \ldots$, and since there are only finitely many points of $Y$ in $\bar{B}$, this process terminates with some $\mathbf{y}_{m}=\overline{\mathbf{y}}$, which gives (4.5).

By hypothesis $\rho \geq 4 R$, hence

$$
\mathbf{y}_{1} \in B\left(\mathbf{y}_{0} ; 2 R\right) \subseteq B\left(\mathbf{y}_{0} ; \rho-2 R\right) .
$$

Thus

$$
\mathbf{y}_{1} \in X \cap B\left(\mathbf{y}_{0} ; \rho-2 R\right) \subseteq X \cap B\left(\mathbf{y}_{0} ; \rho\right),
$$

so Lemma 4.1 applies to give

$$
g \cdot \mathbf{y}_{1} \in \operatorname{ST}_{\mathbf{y}^{\prime}}(X ; \rho) .
$$


Now $g$ is a $(Y, Z ; \rho)$-isometry that takes $\operatorname{ST}_{\mathbf{y}}(X ; \rho)$ to $\operatorname{ST}_{\mathbf{y}^{\prime}}(X ; \rho)$, hence it is a $(Y, Z ; \rho-$ $2 R)$-isometry of $\mathrm{ST}_{\mathbf{y}_{1}}(X ; \rho-2 R)$ to some $\mathrm{ST}_{\mathbf{y}_{1}^{\prime}}(X ; \rho-2 R)$ with $\mathbf{y}_{1}^{\prime} \in Y$. Lemma 4.1 then implies that $g$ is a $(Y, Z ; \rho)$-isometry of $\operatorname{ST}_{\mathbf{y}_{1}}(X ; \rho)$ to $\operatorname{ST}_{\mathbf{y}_{1}^{\prime}}(X ; \rho)$. Since $\operatorname{ST}_{\mathbf{y}_{2}}(X ; \rho-$ $2 R) \subseteq \operatorname{ST}_{\mathbf{y}_{1}}(X ; \rho)$ by Lemma 4.1 we get that $g$ is a $(Y, Z ; \rho-2 R)$ isometry of $\mathrm{ST}_{\mathbf{y}_{2}}(X ; \rho-2 R)$ to some $\mathrm{ST}_{\mathbf{y}_{2}^{\prime}}(X ; \rho-2 R)$. Continuing in this fashion, we eventually obtain that $g$ is a $(Y, Z ; \rho)$ isometry of $\operatorname{ST}_{\overline{\mathbf{y}}}(X ; \rho)$ to some $\operatorname{ST}_{\overline{\mathbf{y}}^{\prime}}(X ; \rho)$. In particular,

$$
g \cdot \overline{\mathbf{y}}=\overline{\mathbf{y}}^{\prime} \in Y \text {. }
$$

By a similar argument $g^{-1} \cdot \overline{\mathbf{y}} \in Y$ for all $\overline{\mathbf{y}} \in Y$, hence $g \in \operatorname{Sym}(Y)$.

Next consider an arbitrary $\overline{\mathbf{z}} \in Z$. Since $Y$ is an $(r, R)$-set, the closed ball $B(\overline{\mathbf{z}} ; R)$ contains some point $\overline{\mathbf{y}} \in Y$. By the argument above $g$ is a $(Y, Z ; \rho)$-isometry of $\operatorname{ST}_{\overline{\mathbf{y}}}(X ; \rho)$ to some $\operatorname{ST}_{\overline{\mathbf{y}}^{\prime}}(X ; \rho)$. Thus the point $\overline{\mathbf{z}}$ is mapped to a point $\overline{\mathbf{z}}^{\prime} \in Z$ in $\operatorname{ST}_{\overline{\mathbf{y}}^{\prime}}(X ; \rho)$, i.e., $g \cdot \overline{\mathbf{z}}=\overline{\mathbf{z}}^{\prime} \in Z$. Similarly $g^{-1} \cdot \overline{\mathbf{z}} \in Z$, so $g \in \operatorname{Sym}(Z)$.

Proof of Theorem 4.1. Partition the Delone set

$$
Y:=\bigcup_{j=1}^{k} Y_{j},
$$

in which the $Y_{j}$ comprise the $(Y, Z ; \rho)$-isometry classes of $\operatorname{ST}_{\mathbf{y}}(Y, Z ; \rho)$ and $k=$ $\left|\operatorname{ST}_{Y}(Y, Z ; \rho)\right|$. For each $\mathbf{y}, \mathbf{y}^{\prime} \in Y_{j}$ there is some $g=g_{\mathbf{y}, \mathbf{y}^{\prime}} \in \operatorname{Sym}\left(\mathbb{E}^{n}\right)$ which is a $(Y, Z ; \rho)$-isometry from $\operatorname{ST}_{\mathbf{y}}(Y, Z ; \rho)$ to $\operatorname{ST}_{\mathbf{y}^{\prime}}(Y, Z ; \rho)$. By Lemma 4.2 each $g_{\mathbf{y}, \mathbf{y}^{\prime}} \in$ $\operatorname{Sym}(Y) \cap \operatorname{Sym}(Z)$. Let $G$ denote the subgroup of $\operatorname{Sym}\left(\mathbb{E}^{n}\right)$ generated by all such $g_{\mathbf{y}, \mathbf{y}^{\prime}}$ for $1 \leq j \leq k$. Then

$$
G \subseteq \operatorname{Sym}(Y) \cap \operatorname{Sym}(Z) \subseteq \operatorname{Sym}(X)
$$

Since $Y$ is a Delone set and $G \subseteq \operatorname{Sym}(Y)$, it follows that $G$ acts discontinuously on $\mathbb{R}^{n}$.

Next, Lemma 3.3 implies that some $Y_{j}$ is an enclosing set. Choose any $\mathbf{y} \in Y_{j}$, and since all $g_{\mathbf{y}, \mathbf{y}^{\prime}} \in G$ for $\mathbf{y}^{\prime} \in Y_{j}$, we have

$$
Y_{j} \subseteq G \cdot \mathbf{y} \subseteq(\operatorname{Sym}(Y) \cap \operatorname{Sym}(Z)) \cdot \mathbf{y} \subseteq \operatorname{Sym}(Y) \cdot \mathbf{y} \subseteq Y .
$$

Then $\operatorname{Sym}(Y) \cap \operatorname{Sym}(Z)$ and $\operatorname{Sym}(Y)$ are both crystallographic groups by Corollary 3.1, and $Y$ is a multiregular point system by Theorem 1.1 .

Finally, since

$$
Y_{j} \subseteq G \cdot \mathbf{y} \subseteq \operatorname{Sym}(X) \cdot \mathbf{y} \subseteq X,
$$

it follows that $\operatorname{Sym}(X)$ is a crystallographic group by Corollary 3.1, and $X$ is a multiregular point system by Theorem 1.1. The number of isomorphism classes of $\left\{\operatorname{ST}_{\mathbf{x}}(X)\right.$ : $\mathbf{x} \in X\}$ is at most $k$, because the action of $\operatorname{Sym}(Y) \cap \operatorname{Sym}(Z)$ on $X$ gives $k$ isomorphism classes by Lemma 4.2, and $\operatorname{Sym}(Y) \cap \operatorname{Sym}(Z) \subseteq \operatorname{Sym}(X)$.

In the remainder of this section we consider the special case $Y=X$ and $Z=\emptyset$, and prove Theorem 1.3 using Corollary 4.1. We begin with the following bound: 
Lemma 4.3. If $X$ is an $(r, R)$-set in $\mathbb{R}^{n}$ and $\mathbf{x} \in X$, then

$$
\left|\operatorname{Lsym}_{\mathbf{x}}(X ; 2 R)\right| \leq\left(\frac{2 R}{r}+1\right)^{n^{2}}
$$

Proof. The point $\mathbf{x} \in X$ is an $R$-point by the proof of Lemma 3.2. In particular, the finite set $\tilde{X}:=X \cap \bar{B}(\mathbf{x} ; 2 R)$ contains $\mathbf{x}$ as an enclosed point, by the proof of Lemma 3.1. We can find $n+1$ points $\mathbf{x}_{0}=\mathbf{x}, \mathbf{x}_{1}, \mathbf{x}_{2}, \ldots, \mathbf{x}_{n} \in \tilde{X}$ which form a full-dimensional simplex. (See the proof of Carathéodory's theorem in [30, (2.2.12)].) Each element $g$ of $\operatorname{Lsym}_{\mathbf{x}}(X ; 2 R)$ fixes $\mathbf{x}$, and is completely determined by specifying the images $g \cdot \mathbf{x}_{i}$ of $\mathbf{x}_{i}$ for $1 \leq i \leq n$. Now

$$
\left\|g \cdot \mathbf{x}_{i}-\mathbf{x}_{0}\right\|=\left\|g \cdot \mathbf{x}_{i}-g \cdot \mathbf{x}_{0}\right\|=\left\|\mathbf{x}_{i}-\mathbf{x}_{0}\right\| \leq 2 R,
$$

hence each image $g \cdot \mathbf{x}_{i}$ lies in $\tilde{X}$. To prove (4.6) it therefore suffices to show that

$$
|\tilde{X}| \leq\left(\frac{2 R}{r}+1\right)^{n}
$$

for there are then at most $(2 R / r+1)^{n^{2}}$ choices for $\left\{g \cdot \mathbf{x}_{i}: 1 \leq i \leq n\right\}$. To prove (4.6), note that since $X$ is an $(r, R)$-set, the open balls $B^{\circ}\left(\mathbf{x}_{i} ; r\right)$ are pairwise disjoint. For if two balls had a common point $\mathbf{w}$, the open ball $B^{\circ}(\mathbf{w} ; r)$ would contain two points of $X$, contradicting the $r$-uniformly discrete property. All these balls lie inside the ball $B(\mathbf{x} ; 2 R+r)$. Comparing the volumes that these balls cover gives

$$
\operatorname{Vol}\left(\bigcup_{\mathbf{x}^{\prime} \in \tilde{X}} B^{\circ}\left(\mathbf{x}^{\prime} ; r\right)\right)=\kappa_{n} r^{n}|\tilde{X}| \leq \kappa_{n}(2 R+r)^{n}=\operatorname{Vol}(B(\mathbf{x} ; 2 R+r)),
$$

in which $\kappa_{n}$ denotes the volume of an $n$-dimensional unit ball, and this yields (4.7).

Proof of Theorem 1.3. We suppose that $X$ is an $(r, R)$-set having the property that all $\rho$-stars $\left\{\operatorname{ST}_{\mathbf{x}}(X ; \rho): \mathbf{x} \in X\right\}$ fall in $k=N_{X}(\rho)$ isometry classes, and that

$$
\rho>2 f(n, r, R) R k
$$

with

$$
f(n, r, R):=\left(n^{2}+1\right) \frac{\log (2 R / r+2)}{\log 2} .
$$

We deduce Theorem 1.3 by showing that for some

$$
\rho_{j}:=2 j R \quad \text { with } \quad 2 \leq j \leq k f(n, r, R),
$$

the two hypotheses:

(a) $N_{X}\left(\rho_{j}\right)=N_{X}\left(\rho_{j}-2 R\right)=k^{\prime} \leq k$; and

(b) $\operatorname{Lsym}_{\mathbf{x}}\left(X ; \rho_{j}\right)=\operatorname{Lsym}_{\mathbf{x}}\left(X ; \rho_{j}-2 R\right)$ for all $\mathbf{x} \in X$; 
simultaneously hold. If so, then the hypothesis of Corollary 4.1 holds for $\rho_{j}$ since $\rho_{j} \geq$ $4 R$, and it implies Theorem 1.3 since $\rho \geq \rho_{j}$.

To proceed, we observe that $N_{X}\left(\rho^{\prime}\right)$ is a nondecreasing function of $\rho^{\prime}$, hence

$$
N_{X}\left(\rho_{j}\right) \leq N_{X}(\rho)=k
$$

Thus $N_{X}\left(\rho_{j}\right)$ can assume at most $k$ different values. Next, the function $\left|\operatorname{Lsym}_{\mathbf{x}}(X ; \rho)\right|$ is a nonincreasing function of $\rho$ by (4.2). If

$$
\left|\operatorname{Lsym}_{\mathbf{x}}\left(X ; \rho_{j}\right)\right| \neq\left|\operatorname{Lsym}_{\mathbf{x}}\left(X ; \rho_{j-1}\right)\right|,
$$

then

$$
\log \left|\operatorname{Lsym}_{\mathbf{x}}\left(X ; \rho_{j}\right)\right| \leq \log \left|\operatorname{Lsym}_{\mathbf{x}}\left(X ; \rho_{j-1}\right)\right|-\log 2,
$$

since a strict subgroup of a finite group $G$ has index at least 2 in $G$.

We now argue by contradiction. So suppose that for each $j$ with $2 \leq j \leq k f(n, r, R)$, at least one of (a) or (b) does not hold for $\rho_{j}$. If condition (a) does not hold for $\rho_{j}$, and $j \geq 3$, then

$$
N_{X}\left(\rho_{j}\right)>N_{X}\left(\rho_{j-1}\right),
$$

so this can happen for at most $k-1$ different values of $j$. To deal with condition (b), we select representatives $\left\{\mathbf{x}_{i}: 1 \leq i \leq k\right\}$ of each of the $k$ isometry classes of $\left\{\operatorname{ST}_{\mathbf{x}}(X ; \rho): \mathbf{x} \in X\right\}$ and define the quantity

$$
I_{j}:=\sum_{i=1}^{k} \log \left|\operatorname{Lsym}_{\mathbf{x}_{i}}\left(X ; \rho_{j}-2 R\right)\right| \quad \text { for } \quad 2 \leq j \leq k f(n, r, R) .
$$

Lemma 3.3 gives

$$
\log \left|\operatorname{Lsym}_{\mathbf{x}}(X ; 2 R)\right| \leq n^{2} \log \left(\frac{2 R}{r}+1\right)
$$

which yields

$$
I_{2} \leq k n^{2} \log \left(\frac{2 R}{r}+1\right)
$$

If (b) does not hold for a given $\rho_{j}$ with $j \geq 3$, then (4.8) applies to at least one of the representative classes $\mathbf{x}_{i}$, hence

$$
I_{j} \leq I_{j-1}-\log 2 .
$$

Since all $I_{j} \geq 0$, this can occur for at most $k n^{2} \log _{2}(2 R / r+1)$ values of $j$. Thus

$$
\#\{j\} \leq k+k n^{2} \log _{2}\left(\frac{2 R}{r}+1\right) .
$$

We obtain a contradiction since

$$
\#\{j\} \geq k f(n, r, R)-1>k+k n^{2} \log _{2}\left(\frac{2 R}{r}+1\right),
$$


which we obtain using

$$
k \log _{2}\left(\frac{2 R}{r}+2\right)>k \log _{2}(4) \geq k+1,
$$

since $R / r>1$ for $n \geq 2$.

Now suppose that $Y$ is a Delone set all of whose $\rho$-stars are isometric to one of $\left\{\mathrm{ST}_{\mathbf{x}}(X ; \rho): \mathbf{x} \in X\right\}$. We reduce to the case that $\mathbf{0} \in Y$ by translating $Y$ to $Y-\mathbf{y}$, if necessary. The proof above produced $\rho_{j}$ with $4 R \leq \rho_{j} \leq \rho$ such that (a) and (b) above hold, and these conditions imply that any $\left(\rho_{j}-2 R\right)$-star of $Y$ extends in a unique way to a $\rho_{j}$-star of $Y$.

By hypothesis

$$
\operatorname{ST}_{\mathbf{0}}\left(Y ; \rho_{j}\right)=Q\left(\operatorname{ST}_{\mathbf{x}_{0}}\left(X ; \rho_{j}\right)-\mathbf{x}_{0}\right),
$$

for some $Q \in O(n, \mathbb{R})$ and $\mathbf{x}_{\mathbf{0}} \in X$. Now each $\mathbf{y} \in Y \cap B\left(\mathbf{0} ; \rho_{j}-2 R\right)$ has $\operatorname{ST}_{\mathbf{y}}\left(Y ; \rho_{j}-\right.$ $2 R) \subseteq \operatorname{ST}_{\mathbf{0}}\left(Y, \rho_{j}\right)$, and this $\left(\rho_{j}-2 R\right)$-star extends uniquely to $\operatorname{ST}_{\mathbf{y}}\left(Y ; \rho_{j}\right)$. The extensions cover all points of $Y$ in the ball $B\left(\mathbf{0} ; \rho_{j}+R\right)$ because $\operatorname{ST}_{\mathbf{0}}\left(Y ; \rho_{j}\right)$ inherits the $(r, R)$-property of $X$ to within distance $R$ of its boundary. Since the uniqueness of these extensions holds for both $X$ and $Y$, we obtain

$$
\operatorname{ST}_{\mathbf{0}}\left(Y ; \rho_{j}\right)=Q\left(\operatorname{ST}_{\mathbf{x}_{0}}\left(X ; \rho_{j}+R\right)-\mathbf{x}_{0}\right) .
$$

Repeating the same argument, by induction on $k \geq 1$, we obtain

$$
\operatorname{ST}_{\mathbf{0}}\left(Y ; \rho_{j}+k R\right)=Q\left(\mathrm{ST}_{\mathbf{x}_{0}}\left(X ; \rho_{j}+k R\right)-\mathbf{x}_{0}\right) \quad \text { all } \quad k \geq 1 .
$$

Thus $Y-\mathbf{y}=Q\left(\mathbf{x}-\mathbf{x}_{0}\right)$, which gives (1.3).

The following examples show that some hypothesis like (1.1) is necessary in Theorem 1.3 to conclude that $X$ is a multiregular point system.

Definition 4.3. A Delone set $X$ has the locally finite atlas property under isometries, or is a Delone set of finite type under isometries, if $|\mathrm{ST}(X ; \rho)|$ is finite for each finite $\rho>0$.

In this case the function

$$
N_{X}(\rho):=|\operatorname{ST}(X ; \rho)|, \quad 0<\rho<\infty,
$$

is a nondecreasing integer-valued function. Theorem 1.3 states that if

$$
N_{X}(\rho)<\frac{\rho}{C R},
$$

for $C=2\left(n^{2}+1\right) \log _{2}(2 R / r+2)$, then $N_{X}(\rho)$ is necessarily a bounded function. The examples below exhibit Delone sets $X$ with $N_{X}(\rho) \rightarrow \infty$ as $\rho \rightarrow \infty$, in one case with $N_{X}(\rho)$ growing linearly in $\rho$.

Many studies of quasicrystalline structures have considered equivalence of neighborhoods under translations rather than under isometries. Let $N_{X}^{*}(\rho)$ denote the number of translation-equivalence classes of $\rho$-stars of $X$. We clearly have

$$
N_{X}(\rho) \leq N_{X}^{*}(\rho) \quad \text { for } \quad \rho>0 .
$$


Definition 4.4. A Delone set $X$ has the locally finite atlas property under translations, or is a Delone set of finite type under translations, if $N_{X}^{*}(\rho)$ is finite for all $\rho>0$.

This property obviously implies the locally finite atlas property under isometries. It is studied in [16].

Example 4.1 (Crystal with "Defects"). Consider the set $X$ in $\mathbb{R}^{2}$ given by

$$
X=\left\{(m, n) \in \mathbb{Z}^{2}: n \neq 0\right\} \cup\{(m+\theta, 0): m \in \mathbb{Z}\},
$$

where $\theta$ is a constant with $0<\theta<\frac{1}{2}$. It is a Delone set with $r=\frac{1}{2}$ and $R=\sqrt{2} / 2$. It is not a multiregular point system, since the row of "defects" along the $x$-axis makes $|\mathrm{ST}(X)|=\infty$. However, $X$ has the locally finite atlas property under translations, with

$$
\lfloor\rho\rfloor \leq N_{X}(\rho)=N_{X}^{*}(\rho) \leq\lfloor\rho\rfloor+2 ;
$$

a complete set of isometry classes of $\rho$-stars have centers contained in the set $\mathbf{x}_{0}=(\theta, 0)$ and $\left\{\mathbf{x}_{i}=(0, i): 1 \leq i \leq\lfloor\rho\rfloor+1\right\}$. Its local stabilizers are

$$
\operatorname{Lsym}_{\mathbf{x}}(X ; \rho)= \begin{cases}\mathbb{Z} / 2 \mathbb{Z} & \text { if } \mathbf{x}=(m+\theta, 0), \\ \{1\} & \text { if } \mathbf{x}=(m, n), \text { with } 1 \leq|n|<\rho, \\ D_{8} & \text { if } \mathbf{x}=(m, n), \text { with }|n| \geq \rho,\end{cases}
$$

in which $D_{8}$ is the symmetry group of the square. For any $k \geq 3$, if $\rho=k-3 / 2$, then $|\mathrm{ST}(X ; k-3 / 2)|=k$. The scaled set $X^{\prime}=\sqrt{2} R X$ is thus an $(r, R)$-set in $\mathbb{R}^{2}$ with $\rho=\sqrt{2} R(k-3 / 2)$ which has $\left|\mathrm{ST}\left(X^{\prime} ; \rho\right)\right|=k$.

There are similar examples in $\mathbb{R}^{n}$. Consider the set $X$ in $\mathbb{R}^{n}$ given by

$$
\begin{aligned}
X= & \left\{\left(m_{1}, \ldots, m_{n}\right) \in \mathbb{Z}^{n}:\left|m_{n}\right|>1\right\} \\
& \cup\left\{\left(m_{1}+\theta, \ldots, m_{n-1}+\theta, 0\right):\left(m_{1}, \ldots, m_{n-1}\right) \in \mathbb{Z}^{n-1}\right\},
\end{aligned}
$$

with $0<\theta<\frac{1}{2}$, which is an $(r, R)$-set with $r=\frac{1}{2}, R=\frac{1}{2} \sqrt{n}$. It is not a multiregular point system, but its group of $\operatorname{symmetries} \operatorname{Sym}(X)$ includes an $(n-1)$-dimensional lattice of translations. The set $X^{\prime}=(2 / \sqrt{n}) R X$ is an $(r, R)$-set that has $\left|\mathrm{ST}\left(X^{\prime} ; \rho\right)\right|=k$ for $\rho=(2 / \sqrt{n}) R\left(k-\frac{3}{2}\right)$.

Example 4.2 (Penrose Tilings). Many constructions proposed in connection with quasicrystals give aperiodic Delone sets $X$ which have the locally finite atlas property under translations. Penrose tilings provide examples. Penrose tilings are tilings of $\mathbb{R}^{2}$ by two types of polygonal tiles called "kites" and "darts," respectively, see [4], [5], [13, p. 539], and [25]. We associate to any Penrose tiling $\mathcal{T}$ a Delone set $X=X(\mathcal{T})$ by choosing a fixed point in each "kite" and "dart." Such tilings have a repetitivity property (under translations), which is that there is a function $L_{X}(\rho)$ such that a translate of each finite configuration ("patch") of tiles of diameter $\rho$ occurs inside any ball of diameter $L_{X}(\rho)$. For Penrose tilings we can take $L_{X}(\rho)=\left(\tau^{5}+2\right) \rho \doteq 13.09 \rho$, where $\tau=(1+\sqrt{5}) / 2$, see Grünbaum and Shephard [13, p. 563]. We show that

$$
N_{X}^{*}(\rho) \leq 830 \rho^{2} \quad \text { for } \quad \rho \geq 3 .
$$


This holds since all "kites" and "darts" that touch a ball of radius $13.09 \rho$ are completely contained in a ball of radius $13.09 \rho+(\tau+1) \leq 14 \rho$ for $\rho \geq 3$, and each "kite" and "dart" has area at least $\frac{3}{4}$. Thus $830 \rho^{2}$ is an upper bound for the number of points of $X$ that lie in any ball of radius $13.09 \rho$, and these points include among them representatives of all translation-equivalence classes of $\rho$-stars.

We can choose the Penrose tiling $\mathcal{T}$ to have no global symmetry, in which case $X=X(\mathcal{T})$ has $\operatorname{Sym}(X)=\{I\}$. If we choose $\rho$ so that $N_{X}(\rho)=k$, then (4.11) implies that $\rho$ grows at least proportionally to $\sqrt{k}$ as $k \rightarrow \infty$.

For other properties of Penrose tilings, see [4], [5], [13], [21], [22], and [25].

Example 4.3 (Pinwheel Tilings). The pinwheel tilings of the plane studied by Radin [20] have the locally finite atlas property under isometries, but do not have the locally finite atlas property under translations. We obtain a Delone set $X$ by choosing a fixed point in each prototile. For the Conway tesselation of the plane described in Section 2 of [20] and [25, Section 7.4], it can be proved that $N_{X}(\rho)$ is finite, with $N_{X}(\rho)=O\left(\rho^{2}\right)$. Radin's results imply that $N_{X}^{*}(\rho)=+\infty$ for $\rho>4 R$.

\section{Acknowledgments}

We are grateful to the Fields Institute of the University of Toronto for its hospitality during the special semester on Long Range Aperiodic Order. The second author thanks P. Shor for a helpful remark.

\section{References}

1. A. Bravais, Mémoire sur les systémes formes par des points distribués regulièrement sur un plan au dans l'espace, J. Ecole Polytechique, 19 (1850), 1-128. (Translation: On the Systems of Points Regularly Distributed on a Plane or in Space, Monograph No. 4, American Crystallographic Association, 1949.)

2. P. Buser, A geometric proof of Bieberbach's theorems on crystallographic groups, L'Enseignement Math., 31 (1985), 137-145.

3. L. Charlap, Bieberbach Groups and Flat Manifolds, Springer-Verlag, New York, 1986.

4. N. G. deBruijn, Algebraic theory of Penrose's non-periodic tilings of the plane I and II, Kon. Nederl. Akad. Wetensch, $\mathbf{A 8 4}$ (1981), 36-66. [Indag. Math., 43 (1981), 38-66.]

5. N. G. deBruijn, Updown generation of Penrose patterns, Indag. Math., N.S., 1 (1990), 201-220.

6. B. N. Delone [B. N. Delaunay], N. P. Dolbilin, M. I. Shtogrin, and R. V. Galiulin, A local criterion for regularity of a system of points, Soviet Math. Dokl., 17, No. 2 (1976), 319-322.

7. N. P. Dolbilin and M. I. Shtogrin, unpublished result, 1976.

8. N. P. Dolbilin and M. I. Shtogrin, A local criterion for a crystal structure, Abstracts of the IX ${ }^{\text {th }}$ All-Union Geometrical Conference, Kishinev, 1988, p. 99 (in Russian).

9. P. Engel, Geometric Crystallography, Reidel, Dordrecht, 1986.

10. P. Engel, Geometric crystallography, In: Handbook of Convex Geometry, Vol. B (P. Gruber and J. M. Wills, Eds.), North-Holland, Amsterdam, 1993, pp. 989-1041.

11. R. V. Galiulin, Axiomatic construction of the geometrical principles of crystallography, Soviet Phys. Cryst., 24, No. 4 (1979), 381-383.

12. R. V. Galiulin, Delaunay systems, Soviet Phys. Cryst., 25, No. 5 (1980), 517-520.

13. B. Grünbaum and G. C. Shephard, Tilings and Patterns, Freeman, New York, 1987. 
14. D. Hilbert and S. Cohn-Vossen, Anschauliche Geometrie, Wiss. Buchgelleshaft, Darmstadt, 1931. [English Translation: Geometry and the Imagination, Chelsea, New York, 1952.]

15. J. C. Lagarias, Meyer's concept of quasicrystal and quasiregular sets, Comm. Math. Phys., 179 (1996), 365-376.

16. J. C. Lagarias, Geometric models for quasicrystals I. Delone sets of finite type, Discrete Comput. Geom., to appear.

17. J. C. Lagarias, Geometric models for quasicrystals II. Local rules under isometries, Discrete Comput. Geom., to appear.

18. J. Milnor, Hilbert's Problem 18: On crystallographic groups, fundamental domains, and on sphere packing, In: Mathematical Developments Arising from Hilbert Problems, Proc. Symp. Proc. Math., Vol. 28, American Mathematical Society: Providence, RI, 1976, pp. 491-506.

19. C. Radin, Global order from local sources, Bull. Amer. Math. Soc. (N.S.), 25 (1991), 335-364.

20. C. Radin, The pinwheel tilings of the plane, Ann. of Math., 139 (1994), 661-702.

21. E. A. Robinson, Jr., The dynamical theory of tilings and quasicrystallography, In: Ergodic Theory of $\mathbb{Z}^{d}$-Actions (M. Pollicott and K. Schmidt, Eds.), London Mathematical Society Lecture Notes, No. 228, Cambridge University Press, Cambridge, 1996, pp. 451-473.

22. E. A. Robinson, Jr., The dynamical properties of Penrose tilings, Trans. Amer. Math Soc., 348 (1996), 4447-4464.

23. R. L. E. Schwarzenberger, N-Dimensional Crystallography, Pitman, London, 1980.

24. M. Senechal, Brief history of geometric crystallography, In: Historical Atlas of Crystallography (J. Limade Ferin, Ed.), Kluwer Academic, Dordrecht, 1990.

25. M. Senechal, Quasicrystals and Geometry, Cambridge University Press, Cambridge, 1995.

26. M. I. Shtogrin, unpublished result, 1977.

27. L. Sohncke, Die regelmässigen ebenen Punktsysteme von unbregrentzer Ausdehnung, J. Reine Angew. Math., 77 (1874), 47-101.

28. L. Sohncke, Entwicklung einer Theorie der Krystallstructur, B. G. Teubner, Leipzig, 1879.

29. L. Sohncke, Erweiterung der Theory der Krystallstructur, Z. Krist., 14 (1888), 426-446.

30. J. Stoer and C. Witzgall, Convexity and Optimization in Finite Dimensions I, Springer-Verlag, Berlin, 1970.

31. A. Vince, Periodicity, quasiperiodicity and Bieberbach's theorem on crystallographic groups, Amer. Math. Monthly, 104 (1997), 27-35.

Received September 13, 1996, and in revised form September 27, 1996, February 6, 1997, and May 7, 1997. 